\title{
Article
}

\section{Basic singular fields in the theory of impulsive supersonic leading-edge noise}

Chapman, C.J. and Powles, Christopher

Available at http://clok.uclan.ac.uk/27997/

Chapman, C.J. and Powles, Christopher ORCID: 0000-0001-9175-2328 (2019) Basic singular fields in the theory of impulsive supersonic leading-edge noise. Wave Motion, 89 . pp. 79-92. ISSN 0165-2125

It is advisable to refer to the publisher's version if you intend to cite from the work. http://dx.doi.org/10.1016/j.wavemoti.2019.03.003

For more information about UCLan's research in this area go to http://www.uclan.ac.uk/researchgroups/ and search for <name of research Group>.

For information about Research generally at UCLan please go to http://www.uclan.ac.uk/research/

All outputs in CLoK are protected by Intellectual Property Rights law, including Copyright law. Copyright, IPR and Moral Rights for the works on this site are retained by the individual authors and/or other copyright owners. Terms and conditions for use of this material are defined in the policies page.

\section{CLoK}

Central Lancashire online Knowledge www.clok.uclan.ac.uk

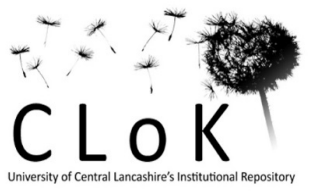




\title{
Basic singular fields in the theory of impulsive supersonic leading-edge noise
}

\author{
C. J. Chapman ${ }^{\mathrm{a}, *}$, C. J. Powles ${ }^{\mathrm{b}}$ \\ ${ }^{a}$ Department of Mathematics, University of Keele, Staffordshire ST5 5BG, UK \\ ${ }^{b}$ School of Physical Sciences and Computing, University of Central Lancashire, Preston, Lancashire PRI 2HE, UK
}

\begin{abstract}
This paper determines the impulsive sound fields produced by sharp-edged gusts striking the leading edge of a supersonic blade or aerofoil. A full three-dimensional theory is provided, so that the gust edges can be at any orientation relative to the blade, and the paper gives complete details of the sound fields produced by gust edges in the spanwise and streamwise directions, and by many combinations of such edges, including corners. The mathematical theory depends on singular sound fields produced by gusts with a delta-function upwash; these are used to derive exact analytical formulae for impusive sound fields of different three-dimensional shapes, and also a Green's function representation of the field which is especially adapted to numerical evaluation. Gusts with top-hat profiles are given particular attention, and also the effect of Gaussian-function smoothing of both delta-function and top-hat profiles. The investigation is complementary to that in a companion paper (Powles \& Chapman 2019) in this special issue of Wave Motion, which determines the smooth sound fields produced by single-frequency gusts. Fourier integration provides the relation between the two types of field.
\end{abstract}

Keywords:

aerofoil, delta-function, gust, impulsive noise, leading edge, supersonic, wavefront

\section{Introduction}

A distinctive feature of a gust is that it can have a sharp edge or be highly localised in the vortex wake of a wing or fan blade, for example, or in random ingested vorticity. In consequence, a gust can produce an impulsive sound field of great intensity when it strikes the leading edge of a downstream blade and is suddenly chopped. The maximum pressure is especially great when the interaction between gust and edge takes place at high speed, as in modern aeroengines. For a subsonic edge, a large body of theory has been worked out, with many three-dimensional impulsive sound fields calculated exactly [1-9]. For a supersonic edge, some three-dimensional impulsive fields have been calculated in [10-13], and a general theory of the generation of such fields is given in [14]. This theory is applied in the special case of two dimensions in [15], and in three dimensions in the PhD thesis [16], but most of the three-dimensional impulsive fields calculated in the thesis are unpublished. The aim of this paper is to present these fields, together with their derivations; a companion paper [17] gives a large number of corresponding non-impulsive fields, produced by single-frequency gusts.

Acoustic fields generated by discontinuous sources display a rich structure of wavefront surfaces. On linear acoustic theory, these propagate (in the appropriate reference frame) at the speed of sound along their normals, and are the dominant geometrical features of the field. In this paper we give explicit formulae for the location and propagation of all such surfaces when they occur, and take care to display them clearly on plots; mathematically, they are represented by Heaviside functions with arguments which are functions of position and time. We give these functions explicitly. A basic reference for the geometry of wavefront surfaces, and for the pressure field nearby, is [18]. Taking the present paper and the companion paper [17] together, one cannot fail to notice the enduring significance of the research in wave theory carried out by J. B. Keller and F. G. Friedlander in the fifteen years or so up about 1960.

\footnotetext{
${ }^{*}$ Corresponding author

Email addresses: c.j.chapman@keele.ac.uk (C. J. Chapman ), cpowles@uclan.ac.uk (C. J. Powles)
} 


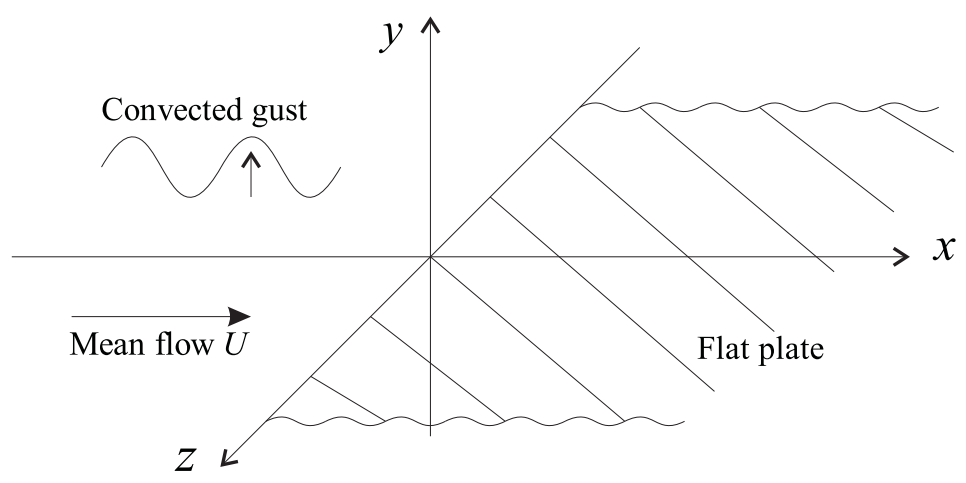

Figure 1: Gust convected at speed $U$ in the $x$-direction past a stationary flat-plate aerofoil. The aerofoil occupies the half-plane $y=0, x \geq 0$, and the leading edge lies along the $z$-axis.

This paper is organised as follows. After setting out the basic equations in $\S 2$, we consider gusts containing a spanwise delta-function in $\S 3$, a streamwise delta-function in $\S 4$, and a point delta-function in $\S 5$. The last of these gives a Green's function representation of a general sound field in a form useful for numerical evaluation. In $\$ 6$ we consider gusts with a corner in the upwash, and hence calculate the field produced by a rectangular gust. In $\$ 7$ we determine the effect of Gaussian-function smoothing of delta-function and top-hat profiles. Conclusions and a historical perspective are presented in $\S 8$.

\section{Fundamental integrals}

As in [17], we consider a flat-plate aerofoil of infinite span occupying the half-plane $y=0, x>0$ in a coordinate system $(x, y, z)$ for which the downstream direction is $x$, the vertical direction is $y$, and the span direction is $z$. There is a mean flow at speed $U$ in the positive $x$ direction, as shown in Figure 1, so that any gust convected towards the aerofoil is chopped at the $z$-axis, i.e. at the aerofoil leading edge. The Mach number of the flow is $M=U / c_{0}>1$, where $c_{0}$ is the speed of sound in the fluid, and we use Doppler-adjusted coordinates

$$
\bar{x}=x /\left(M^{2}-1\right), \quad \bar{y}=y /\left(M^{2}-1\right)^{1 / 2}, \quad \bar{z}=z /\left(M^{2}-1\right)^{1 / 2} .
$$

The gust upwash, i.e. the vertical component of the gust velocity in the plane of the aerofoil, is represented by the function $f(t-x / U, z)$, where $t$ denotes time; this corresponds to convection of the function $f(t, z)$ at speed $U$ in the positive $x$-direction. The Fourier transform of the upwash at the leading edge $x=0$ is

$$
F(\omega, m)=\int_{-\infty}^{\infty} \int_{-\infty}^{\infty} f(t, z) \mathrm{e}^{\mathrm{i}(\omega t-m z)} \mathrm{d} t \mathrm{~d} z
$$

where $\omega$ is the frequency and $m$ is the spanwise wavenumber. The acoustic pressure $p(x, y, z, t)$ due to the gust is

$$
p(x, y, z, t)=\frac{-\rho_{0} M c_{0} \operatorname{sgn}(y)}{(2 \pi)^{2}\left(M^{2}-1\right)^{1 / 2}} \int_{-\infty}^{\infty} \int_{-\infty}^{\infty} F(\omega, m) \mathrm{e}^{-\mathrm{i} \omega\left(t-M \bar{x} / c_{0}\right)} \mathrm{e}^{\mathrm{i} m z} J_{0}\left(\left\{\omega^{2} / c_{0}^{2}+m^{2}\left(M^{2}-1\right)\right\}^{1 / 2} \bar{r}_{h}\right) \mathrm{d} m \mathrm{~d} \omega
$$

where $J_{0}$ is the Bessel function of order zero, $\bar{r}_{h}=\left(\bar{x}^{2}-\bar{y}^{2}\right)^{1 / 2}$ is a cylindrical coordinate measuring hyperbolic distance from the leading edge, and the integration contour in $\omega$ passes above any singularities in the integrand. Eqn. (3) applies in the Mach wedge $|\bar{y}| \leq \bar{x}$ emanating downstream from the leading edge of the aerofoil; elsewhere there is no sound. Reference [14] derives (3) from the convected wave equation and boundary conditions of the problem, and also derives the far-field approximation $p \simeq p_{+}+p_{-}$where

$$
p_{ \pm}(x, y, z, t)=\frac{-\rho_{0} M c_{0} \operatorname{sgn}(y)}{(2 \pi)^{2}\left(M^{2}-1\right)} \frac{1}{\bar{R}_{h}} \int_{-\infty}^{\infty} F\left(\omega, \pm \frac{\omega}{c_{0}}\left(M^{2}-1\right)^{-1 / 2} \tan \bar{\theta}_{h}\right) \mathrm{e}^{-\mathrm{i} \omega\left(t-M \bar{x} / c_{0} \mp \bar{R}_{h} / c_{0}\right)} \mathrm{d} \omega .
$$


Here $\bar{R}_{h}=\left(\bar{x}^{2}-\bar{y}^{2}-\bar{z}^{2}\right)^{1 / 2}$ is a hyperbolic polar distance from the origin, and $\bar{\theta}_{h}$ is a hyperbolic angle relative to the vertical plane $z=0$, defined so that $\tan \bar{\theta}_{h}=-\bar{z} / \bar{R}_{h}$. Eqn. (4) assumes that the upwash is of bounded spanwise extent about the origin and the Fourier transform $F(\omega, m)$ has no singularities in the complex $m$ plane; it applies in the Mach cone $\left|\bar{y}^{2}+\bar{z}^{2}\right|^{1 / 2} \leq \bar{x}$, outside of which there is no far field, given that the upwash is localised. Mach wedges and cones extend downstream of points on the leading edge, with width increasing downstream. The quantities $\bar{r}_{h}$ and $\bar{R}_{h}$ are always real; terms containing them in formulae should be put equal to zero at positions which would make them imaginary, i.e. outside of the corresponding wedge or cone. The right-hand sides of Eqns. (3) and (4) are the fundamental integrals required in this paper.

\section{Spanwise delta-function gusts}

We consider gusts with upwash function

$$
f(t-x / U, z)=f_{0}(t-x / U) \delta(z / a)
$$

in which the function $f_{0}$, representing an arbitrary streamwise variation of upwash velocity, has Fourier transform

$$
F_{0}(\omega)=\int_{-\infty}^{\infty} f_{0}(t) \mathrm{e}^{\mathrm{i} \omega t} \mathrm{~d} t
$$

at the leading edge $x=0$. The term $\delta(z / a)$ localises the gust at span position $z=0$; it is convenient to include the length-scale $a$ in the argument of the delta function, so that integrals with respect to $z$ have the correct dimensions when $f_{0}$ is regarded as a velocity component. The gust strength is the integral of the upwash over the leading edge, and is $a f_{0}(t)$.

Evaluation of the Fourier transform (2) gives $F(\omega, m)=a F_{0}(\omega)$, and then integration with respect to $m$ in (3) gives

$$
p(x, y, z, t)=\frac{-\rho_{0} M c_{0} \bar{v}_{0} \operatorname{sgn}(y)}{2 \pi^{2}} \frac{\bar{a}}{\bar{R}_{h}} \int_{-\infty}^{\infty} F_{0}(\omega) \mathrm{e}^{-\mathrm{i} \omega\left(t-M \bar{x} / c_{0}\right)} \cos \left(\frac{\omega \bar{R}_{h}}{c_{0}}\right) \mathrm{d} \omega .
$$

The bars on $\bar{v}_{0}$ and $\bar{a}$ indicate that Doppler factors have been applied according to the rule implicit in (1), that streamwise quantities are to be divided by $M^{2}-1$, and transverse quantities (vertical and spanwise) are to be divided by $\left(M^{2}-1\right)^{1 / 2}$. Thus $\bar{v}_{0}=v_{0} /\left(M^{2}-1\right)^{1 / 2}$ and $\bar{a}=a /\left(M^{2}-1\right)^{1 / 2}$. This convention is adopted throughout the paper. Expression (7) applies in the Mach cone $\left|\bar{y}^{2}+\bar{z}^{2}\right|^{1 / 2} \leq \bar{x}$, where $\bar{R}_{h}$ is real, and elsewhere the pressure is zero, according to our convention for interpreting expressions containing the quantity $\bar{R}_{h}$.

If the cosine in (7) is written as the sum of two exponentials, the integration with respect to $\omega$ inverts the Fourier transform of $f_{0}$ at arguments

$$
t_{ \pm}=t-\frac{M \bar{x}}{c_{0}} \pm \frac{\bar{R}_{h}}{c_{0}}
$$

to give

$$
p(x, y, z, t)=\frac{-\rho_{0} M c_{0} \bar{v}_{0} \operatorname{sgn}(y)}{2 \pi} \frac{\bar{a}}{\bar{R}_{h}}\left\{f_{0}\left(t_{+}\right)+f_{0}\left(t_{-}\right)\right\} .
$$

Except for the sign dependence on $y$, the field is cylindrically symmetric about the $x$-axis. The field is singular on the Mach cone $\bar{R}_{h}=0$. Expression (9) is a wavefront representation of the sound field, because the quantities $t_{ \pm}$ represent the downstream-facing and upstream-facing parts of a convected sphere, generated with zero radius at the point $z=0$ on the leading edge and then expanding at the speed of sound. This follows from (8), because $t_{ \pm}=t_{0}$ gives the spherical wavefront

$$
\left(x-U\left(t-t_{0}\right)\right)^{2}+y^{2}+z^{2}=c_{0}^{2}\left(t-t_{0}\right)^{2},
$$

which touches the Mach cone $\bar{R}_{h}=0$ on the circular section at $x=\left(\left(M^{2}-1\right) / M^{2}\right) U\left(t-t_{0}\right)$; hence $t_{+}$and $t_{-}$describe the parts of the wavefront downstream and upstream, respectively, of this circle. Note that this circle is upstream of, and of smaller radius than, the equatorial circle at $x=U\left(t-t_{0}\right)$ on the sphere, which has radius $c_{0}\left(t-t_{0}\right)$. 


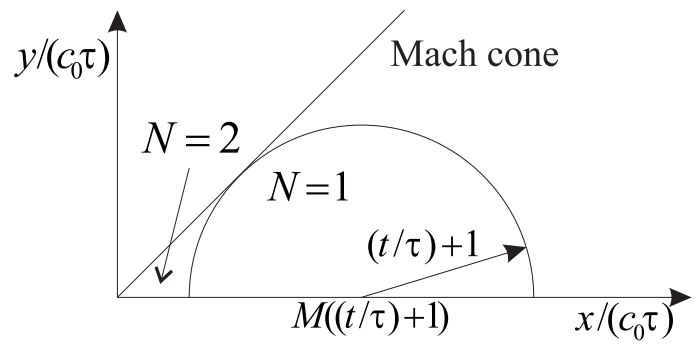

(a)

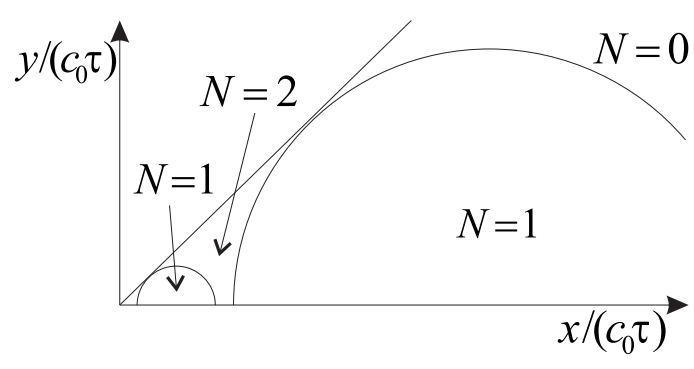

(c)

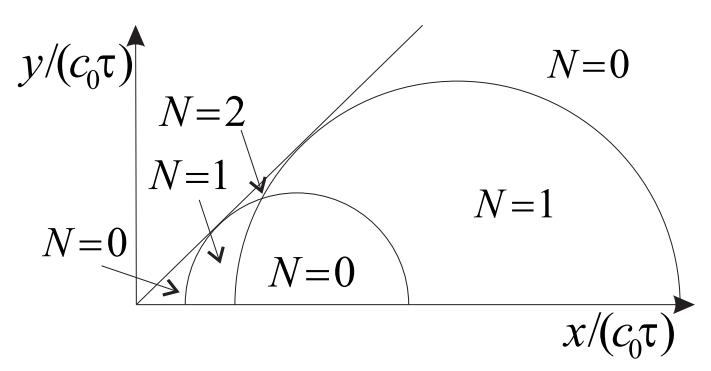

(e)

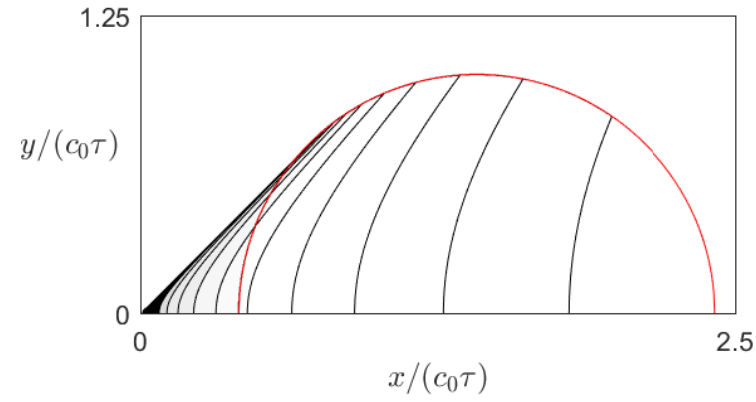

(b)

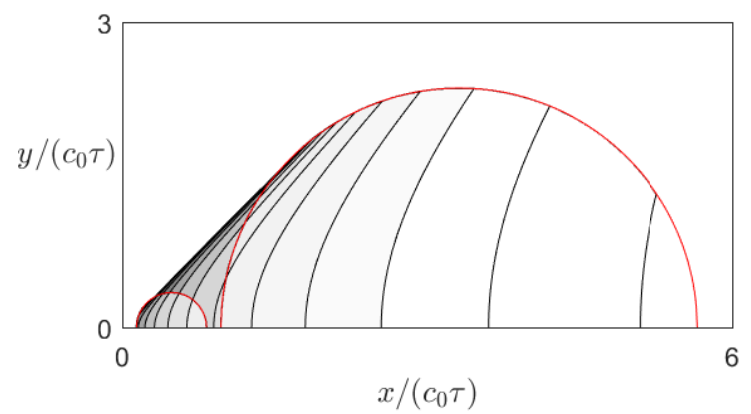

(d)

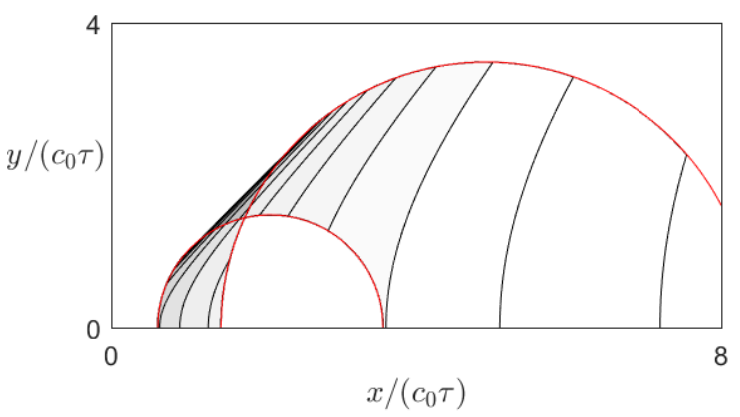

(f)

Figure 2: Development in time of the pressure field generated by a gust with spanwise delta-function and streamwise top-hat profile, convected at Mach number $M=\sqrt{2}$. On the left are wavefronts and corresponding values of $N$; on the right are contours of the pressure field scaled by $\rho_{0} M c_{0} \bar{v}_{0}$, together with the wavefronts marked in red. The pressure is negative or zero everywhere, and the most negative pressures are indicated by the darkest shading. Across the wavefronts, the pressure field is discontinuous. In (a-b), for $t / \tau=0$, the gust is still striking the leading edge; in (c-d), for $t / \tau=0.95 M$, the gust has just passed the leading edge; and in (e-f), for $t / \tau=1.75 M$, the initial and final wavefronts have overlapped, leaving an interior silent region. The plots are obtained from Eqn. (12). 


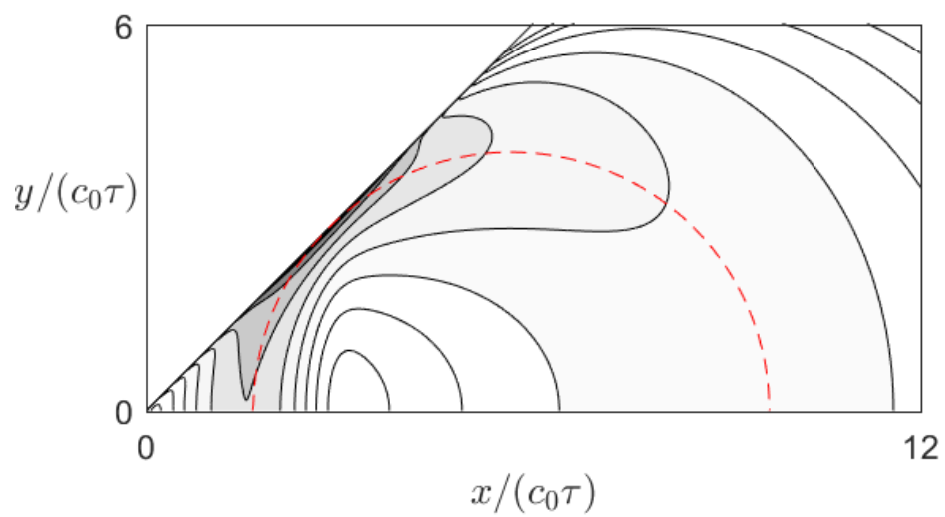

Figure 3: Contours of the pressure field (15) produced by a gust with spanwise delta-function and streamwise Gaussian profile, for $M=\sqrt{2}$. The plot is for $t=4 \tau$, and the pressure has been scaled by $\rho_{0} M c_{0} \bar{v}_{0}$. As in Figure 2, the pressure is negative or zero everywhere, and the darkest shading indicates the most negative pressures. An interior region similar to that in Figure 2(f) is evident, but it has an exponentially small sound field, rather than being completely silent. The dashed red circle is the wavefront $t_{ \pm}=0$ produced at time $t_{0}=0$, in accordance with the wavefront equation (10).

\subsection{The streamwise top-hat gust}

In the upwash function (5) we now take

$$
f_{0}(t-x / U)=v_{0} \mathrm{H}((t-x / U) / \tau,-1,1),
$$

where the top-hat function $\mathrm{H}\left(s, s_{0}, s_{1}\right)$ takes the value 1 for $s_{0}<s<s_{1}$, and zero elsewhere. Thus the upwash at fixed $x$ is non-zero for a time interval of duration $2 \tau$. From (9), the pressure field generated by this upwash is

$$
p(x, y, z, t)=\frac{-\rho_{0} M c_{0} \bar{v}_{0} \operatorname{sgn}(y)}{2 \pi} \frac{\bar{a}}{\bar{R}_{h}} N,
$$

where $N$ is an integer defined by

$$
N=\mathrm{H}\left(t_{+} / \tau,-1,1\right)+\mathrm{H}\left(t_{-} / \tau,-1,1\right) .
$$

At any time $t$, space is divided up into regions bounded by the two spheres $t_{ \pm}=\tau$ and $t_{ \pm}=-\tau$ of the form (10) with $t_{0}=\tau$ and $t_{0}=-\tau$. The possible values $N=0,1,2$ are determined by position relative to these two spheres. For large enough times, the two spheres intersect; then $N=0$ in the region interior to both spheres, so that the pressure field has an interior silent zone. This is illustrated in figure 2, which shows the development of the field in time for $M=\sqrt{2}$. An interior silent zone for an intercepted cylindrical jet was identified in [10].

\subsection{The streamwise Gaussian gust}

A smoother upwash than the top-hat is the Gaussian form

$$
f_{0}(t-x / U)=v_{0} \mathrm{e}^{-((t-x / U) / \tau)^{2} / 2}
$$

which by (9) generates the pressure field

$$
p(x, y, z, t)=\frac{-\rho_{0} M c_{0} \bar{v}_{0} \operatorname{sgn}(y)}{2 \pi} \frac{\bar{a}}{\bar{R}_{h}}\left(\mathrm{e}^{-\left(t_{+} / \tau\right)^{2} / 2}+\mathrm{e}^{-\left(t_{-} / \tau\right)^{2} / 2}\right) .
$$

This is plotted in figure 5.2 for $M=\sqrt{2}$. The pressure field has an exponentially small precursor and tail, as opposed to regions where the field is switched off completely. 


\begin{tabular}{|c|c|c|}
\hline$N=0$ & $N=1 N^{N}$ & $N=1$ \\
\hline & $-\bar{a}$ & $\bar{a}$ \\
\hline
\end{tabular}

(a)

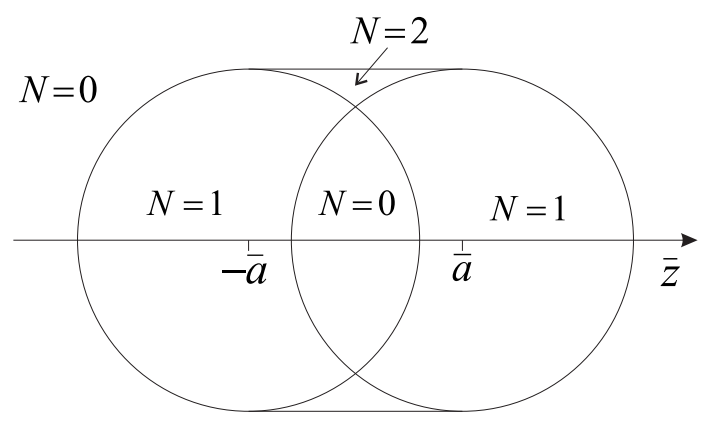

(b)

Figure 4: Regions of causality for a gust with streamwise delta-function and spanwise top-hat profile, for $M=\sqrt{2}$. Downstream vertical sections parallel to the leading edge are shown at times (a) before and (b) after the spherical wavefronts from the ends of the gust have met. Values of $N$ are determined by Eqn. (23). The interior silent region $N=0$ in (b) expands steadily, but the adjacent regions $N=1$ maintain their width; hence at large times, most of space is silent, but impulsive noise propagates in a relatively narrow shell.

\section{Streamwise delta-function gusts}

We now consider the upwash function

$$
f(t-x / U, z)=v_{0} \delta((t-x / U) / \tau) g(z)
$$

where $g(z)$ represents an arbitrary spanwise variation of upwash. Thus sound is generated only at the single instant $t=0$ when the delta-function strikes the leading edge $x=0$, but at this instant the sources may be distributed along the whole of the leading edge in any way. An analysis similar to that of $\S 3$, but with (3) integrated first with respect to $\omega$, leads us to introduce the quantities

$$
z_{ \pm}=z \pm \Delta
$$

representing spanwise positions offset from $z$ by an amount

$$
\Delta=\left\{\left(c_{0} t\right)^{2}-(x-U t)^{2}-y^{2}\right\}^{1 / 2} .
$$

The offset $\Delta$ is defined in in a cylinder of radius $c_{0} t$ parallel to the leading edge and centred on the line $(x, y)=(U t, 0)$; outside of this cylinder, which has equation $\Delta=0$, any expression containing $\Delta$ in a formula is put equal to zero. The Doppler-adjusted value of the offset is $\bar{\Delta}=\Delta /\left(M^{2}-1\right)^{1 / 2}$. In terms of $z_{ \pm}$and $\bar{\Delta}$, the pressure field due to the upwash (16) is

$$
p(x, y, z, t)=\frac{-\rho_{0} M c_{0} \bar{v}_{0} \operatorname{sgn}(y)}{2 \pi} \frac{c_{0} \tau}{\bar{\Delta}}\left\{g\left(z_{+}\right)+g\left(z_{-}\right)\right\} .
$$

Thus the quantities $z_{ \pm}$play the role taken by $t_{ \pm}$in $\S 3$, and similarly $c_{0} \tau / \bar{\Delta}$ takes the place of $\bar{a} / \bar{R}_{h}$. Expression (19) is a wavefront representation of the sound field, in which $z_{ \pm}$refer to the parts of the wavefront facing in the (positive, negative) span directions. The values $z_{ \pm}=z_{0}$ give the spherical wavefront centred on $\left(U t, 0, z_{0}\right)$, i.e.

$$
(x-U t)^{2}+y^{2}+\left(z-z_{0}\right)^{2}=\left(c_{0} t\right)^{2} .
$$




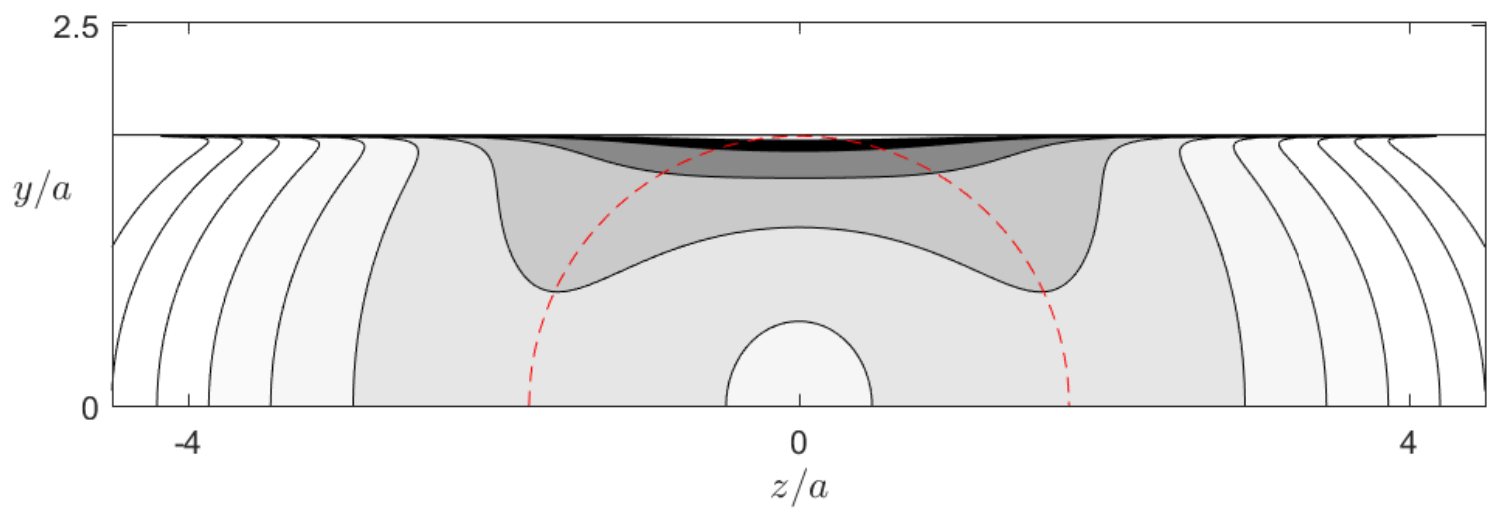

Figure 5: Contours of the pressure field (25) produced by a gust with streamwise delta-function and spanwise Gaussian profile, for $M=\sqrt{2}$. The plot is in the vertical plane $x=U t / 2$ at time $t=2.5 a / c_{0}$, and the pressure has been scaled by $\rho_{0} M c_{0} \bar{v}_{0}$. In the upper half-plane shown, the pressure is negative or zero, and the darkest shading indicates the most negative pressures. The dashed red circle is the wavefront generated by the peak leading-edge upwash, i.e. for $z=0, t=0$.

\subsection{The spanwise top-hat gust}

We now consider the top-hat gust shape

$$
g(z)=\mathrm{H}(z / a,-1,1)
$$

for which the upwash is non-zero only on a length $2 a$ of the leading edge. From (19), the pressure field is

$$
p(x, y, z, t)=\frac{-\rho_{0} M c_{0} \bar{v}_{0} \operatorname{sgn}(y)}{2 \pi} \frac{c_{0} \tau}{\bar{\Delta}} N
$$

where $N$ is the integer

$$
N=\mathrm{H}\left(z_{+} / a,-1,1\right)+\mathrm{H}\left(z_{-} / a,-1,1\right) .
$$

Thus space is divided up into regions bounded by the two expanding spheres $z_{ \pm}=a$ and $z_{ \pm}=-a$, and by the expanding cylinder $\Delta=0$. In each region, $N$ takes a particular value 0,1 , or 2 , so that the field is everywhere a multiple of the field generated by the two-dimensional gust $g(z)=1$, derived in [15]. The evolution of the wavefront geometry is shown in Figure 4, giving vertical sections through the axis of the expanding cylinder at two different times. In (a), the spherical wavefronts from the edges $z= \pm a$ of the gust are still separated; in (b), they have crossed, producing an interior silent region $N=0$.

\subsection{The spanwise Gaussian gust}

The Gaussian form of (16) is obtained by taking

$$
g(z)=\mathrm{e}^{-(z / a)^{2} / 2},
$$

so that by (19) the pressure field is

$$
p(x, y, z, t)=\frac{-\rho_{0} M c_{0} \bar{v}_{0} \operatorname{sgn}(y)}{2 \pi} \frac{c_{0} \tau}{\bar{\Delta}}\left\{\mathrm{e}^{-\left(z_{+} / a\right)^{2} / 2}+\mathrm{e}^{-\left(z_{-} / a\right)^{2} / 2}\right\} .
$$

The field is concentrated near the maxima $z_{ \pm}=0$ of the Gaussian functions, i.e. the sphere of radius $c_{0} t$ centred on $(U t, 0,0)$, and tends to infinity where this sphere touches the cylinder $\Delta=0$ in the vertical plane $z=0$. The field is plotted in Figure 5 for $M=\sqrt{2}$. 


\section{The delta-function gust}

The most basic singular field is that produced by a gust having a delta-function profile in both the stream and the span direction, i.e. with upwash function

$$
f(t-x / U, z)=v_{0} \delta((t-x / U) / \tau) \delta(z / a) .
$$

Since both (9) and (19) apply, the pressure field may be written in either of the forms

$$
p(x, y, z, t)=\frac{-\rho_{0} M c_{0} \bar{v}_{0} \operatorname{sgn}(y)}{2 \pi} \frac{\bar{a}}{\bar{R}_{h}}\left\{\delta\left(t_{+} / \tau\right)+\delta\left(t_{-} / \tau\right)\right\}
$$

or

$$
p(x, y, z, t)=\frac{-\rho_{0} M c_{0} \bar{v}_{0} \operatorname{sgn}(y)}{2 \pi} \frac{c_{0} \tau}{\bar{\Delta}}\left\{\delta\left(z_{+} / a\right)+\delta\left(z_{-} / a\right)\right\} .
$$

These are both equivalent to

$$
p(x, y, z, t)=\frac{-\rho_{0} M c_{0} v_{0} \operatorname{sgn}(y)}{\pi} c_{0} a \tau \delta\left((x-U t)^{2}+y^{2}+z^{2}-\left(c_{0} t\right)^{2}\right) .
$$

The last of these shows explicitly that the pressure field is a delta function localised on a sphere expanding at the speed of sound $c_{0}$ and convected with the free stream at speed $U$. The field is generated at time $t=0$ as a sphere of zero radius at $(x, y, z)=(0,0,0)$, i.e. at the point where the gust strikes the leading edge.

\subsection{Green's function}

If we consider a delta-function unit upwash localised at $t=t_{0}$ and $z=z_{0}$, where $t_{0}$ and $z_{0}$ are arbitrary, we may obtain an arbitrary upwash on multiplying by $f\left(t_{0}, z_{0}\right)$ and integrating with respect to $t_{0}$ and $z_{0}$. Since the problem we are solving is linear, equation (29) together with the identity

$$
v_{0} a \delta\left((x-U t)^{2}+y^{2}+z^{2}-\left(c_{0} t\right)^{2}\right)=\bar{v}_{0} \bar{a} \delta\left(\left(c_{0} t-M \bar{x}\right)^{2}+\bar{z}^{2}-\bar{r}_{h}^{2}\right)
$$

gives a Green's function representation of the pressure field in the form

$$
p(x, y, z, t)=\frac{-\rho_{0} M c_{0} \operatorname{sgn}(y)}{\pi} \int_{-\infty}^{t} \int_{-\infty}^{\infty} f\left(t_{0}, z_{0}\right) \delta\left(\left\{c_{0}\left(t-t_{0}\right)-M \bar{x}\right\}^{2}+\left(\bar{z}-\bar{z}_{0}\right)^{2}-\bar{r}_{h}^{2}\right) c_{0} \mathrm{~d} t_{0} \mathrm{~d} \bar{z}_{0} .
$$

A change of integration variables to $(r, \theta)$ defined by $c_{0} t_{0}=c_{0} t-M \bar{x}+r \sin \theta$ and $\bar{z}_{0}=\bar{z}+r \cos \theta$, where $0<r<\infty$ and $0<\theta<2 \pi$, gives an $r$ integral which can be performed analytically, to leave

$$
p(x, y, z, t)=\frac{-\rho_{0} M c_{0} \operatorname{sgn}(y)}{2 \pi\left(M^{2}-1\right)^{1 / 2}} \int_{0}^{2 \pi} f\left(t-\frac{M \bar{x}}{c_{0}}+\frac{\bar{r}_{h} \sin \theta}{c_{0}}, z+\left(M^{2}-1\right)^{1 / 2} \bar{r}_{h} \cos \theta\right) \mathrm{d} \theta .
$$

This expression is convenient for numerical integration, as it has a finite range of integration and is free of singularities.

The correctness of (32) may be checked by direct integration for upwash $v_{0} \mathrm{e}^{-\mathrm{i} \omega_{0}(t-x / U)}$ and $v_{0} \mathrm{e}^{-\mathrm{i} \omega_{0}(t-x / U)} \delta(z / a)$. These give

$$
p(x, y, z, t)=-\rho_{0} M c_{0} \bar{v}_{0} \operatorname{sgn}(y) \mathrm{e}^{-\mathrm{i} \omega_{0}\left(t-M \bar{x} / c_{0}\right)} \mathrm{J}_{0}\left(\frac{\omega_{0} \bar{r}_{h}}{c_{0}}\right)
$$

and

$$
p(x, y, z, t)=\frac{-\rho_{0} M c_{0} \bar{v}_{0} \operatorname{sgn}(y)}{\pi} \mathrm{e}^{-\mathrm{i} \omega_{0}\left(t-M \bar{x} / c_{0}\right)} \frac{\bar{a}}{\bar{R}_{h}} \cos \left(\frac{\omega_{0} \bar{R}_{h}}{c_{0}}\right),
$$

respectively, in agreement with [17]. 


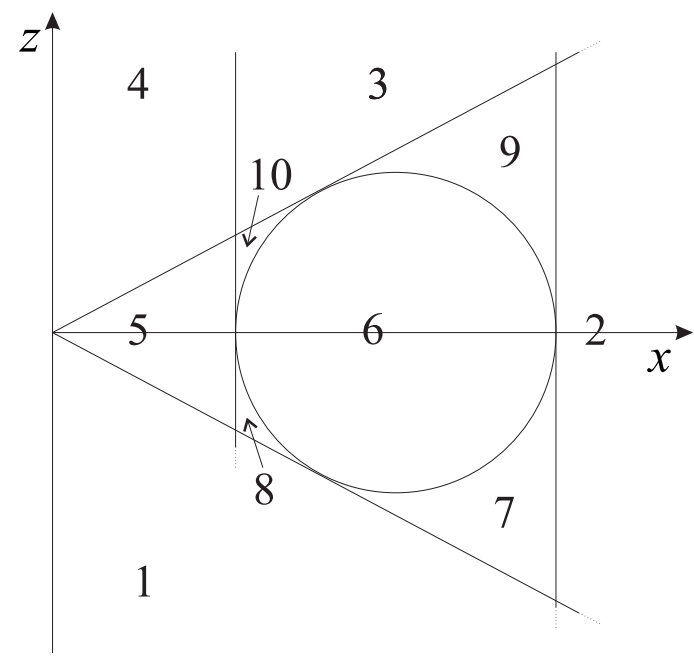

(a)

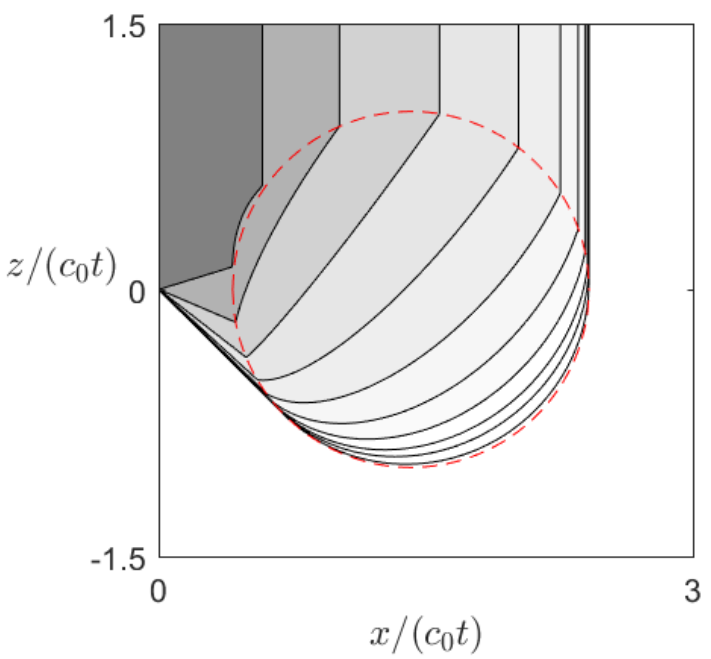

(b)

Figure 6: Pressure field produced by a corner gust for $M=\sqrt{2}$. (a) Division of space into regions separated by wavefronts, shown in section on the aerofoil surface $y=0$. The numbering of the regions corresponds to that in table 1. (b) Contours of pressure on the upper surface of the aerofoil, $y=0^{+}$. The pressure is negative or zero, and the darkest shading indicates the most negative pressures. The dashed red circle is the wavefront generated at the instant the corner of the gust strikes the leading edge.

\section{Corner and rectangular gusts}

A corner gust has upwash

$$
f(t-x / U, z)=v_{0} \mathrm{H}(t-x / U) \mathrm{H}(z),
$$

which is uniform and positive in the quarter plane defined by $x<U t$ and $z>0$, and zero elsewhere. The corner of the gust strikes the leading edge $x=0$ of the aerofoil at span position $z=0$ at time $t=0$. As the gust has no length scale, the pressure field depends on position and time only in the combinations $x /\left(c_{0} t\right), y /\left(c_{0} t\right)$, and $z /\left(c_{0} t\right)$, so that for $t>0$ the field evolves in scale but not shape.

The pressure integral (32) for the gust (35) is

$$
p(x, y, z, t)=\frac{-\rho_{0} M c_{0} \bar{v}_{0} \operatorname{sgn}(y)}{2 \pi} \int_{0}^{2 \pi} \mathrm{H}\left(t-\frac{M \bar{x}}{c_{0}}+\frac{\bar{r}_{h} \sin \theta}{c_{0}}\right) \mathrm{H}\left(\bar{z}+\bar{r}_{h} \cos \theta\right) \mathrm{d} \theta .
$$

Here the Heaviside functions switch between 0 and 1 when $\theta$ passes through a value satisfying $\sin \theta=\left(M \bar{x}-c_{0} t\right) / \bar{r}_{h}$ or $\cos \theta=-\bar{z} / \bar{r}_{h}$, and a switch may or may not occur in the range of integration 0 to $2 \pi$. Hence space is divided into a number of regions, separated by wavefronts, in which the pressure field takes different forms. Figure 6(a) shows a cross-section of these regions in the horizontal plane $y=0$, and table 1 lists the defining properties of these regions. The boundaries are the Mach cone with apex at the origin, the cylindrical surface $\left|M \bar{x}-c_{0} t\right|=\bar{r}_{h}$, which corresponds to the wavefront generated by a sharp-edged two-dimensional gust striking the leading edge at $t=0$, and the spherical surface $\left|M \bar{x}-c_{0} t\right|=\bar{R}_{h}$, which corresponds to the wavefront generated at the origin at $t=0$. The regions do not change in form, but expand at speed $c_{0}$.

The pressure field in region $n$ will be denoted $p_{n}$. In regions 1 and 2, at least one Heaviside function is 0 for all $\theta$, so that $p_{1}=p_{2}=0$. In region 3, the first Heaviside function in (32) switches at two values of $\theta$, to give

$$
p_{3}=\frac{-\rho_{0} M c_{0} \bar{v}_{0} \operatorname{sgn}(y)}{\pi}\left(\frac{\pi}{2}-\sin ^{-1}\left(\frac{M \bar{x}-c_{0} t}{\bar{r}_{h}}\right)\right) .
$$




\begin{tabular}{|c|c|c|}
\hline Region & Streamwise properties & Spanwise properties \\
\hline 1 & & $|\bar{z}|>\bar{r}_{h} ; \bar{z}<0$ \\
2 & $\left|M \bar{x}-c_{0} t\right|>\bar{r}_{h} ; M \bar{x}-c_{0} t>0$ & \\
3 & $\left|M \bar{x}-c_{0} t\right|<\bar{r}_{h}$ & $|\bar{z}|>\bar{r}_{h} ; \bar{z}>0$ \\
4 & $\left|M \bar{x}-c_{0} t\right|>\bar{r}_{h} ; M \bar{x}-c_{0} t<0$ & $|\bar{z}|>\bar{r}_{h} ; \bar{z}>0$ \\
5 & $\left|M \bar{x}-c_{0} t\right|>\bar{r}_{h} ; M \bar{x}-c_{0} t<0$ & $|\bar{z}|<\bar{r}_{h}$ \\
6 & $\left|M \bar{x}-c_{0} t\right|<\bar{R}_{h}$ & $|\bar{z}|<\bar{r}_{h}$ \\
7 & $\bar{r}_{h}>\left|M \bar{x}-c_{0} t\right|>\bar{R}_{h} ; M \bar{x}-c_{0} t>0$ & $|\bar{z}|<\bar{r}_{h} ; \bar{z}<0$ \\
8 & $\bar{r}_{h}>\left|M \bar{x}-c_{0} t\right|>\bar{R}_{h} ; M \bar{x}-c_{0} t<0$ & $|\bar{z}|<\bar{r}_{h} ; \bar{z}<0$ \\
9 & $\bar{r}_{h}>\left|M \bar{x}-c_{0} t\right|>\bar{R}_{h} ; M \bar{x}-c_{0} t>0$ & $|\bar{z}|<\bar{r}_{h} ; \bar{z}>0$ \\
10 & $\bar{r}_{h}>\left|M \bar{x}-c_{0} t\right|>\bar{R}_{h} ; M \bar{x}-c_{0} t<0$ & $|\bar{z}|<\bar{r}_{h} ; \bar{z}>0$ \\
\hline
\end{tabular}

Table 1: Properties of the regions shown in figure 6(a) for the corner gust, required for the analysis of the pressure integral (36).

This matches the field for a two-dimensional top-hat gust (see [15]) while the gust is still striking the leading edge. In region 4, both Heaviside functions take the value 1, so that $p_{4}=-\rho_{0} M c_{0} \bar{v}_{0} \operatorname{sgn}(y)$. In region 5, the second Heaviside function in (32) switches at two values of $\theta$, to give

$$
p_{5}=\frac{-\rho_{0} M c_{0} \bar{v}_{0} \operatorname{sgn}(y)}{\pi} \cos ^{-1}\left(-\frac{\bar{z}}{\bar{r}_{h}}\right) .
$$

Regions 6-10 are more complicated, as both Heaviside functions switch, and it is necessary to determine the range of $\theta$ in which they are non-zero simultaneously. In region 6 the result is

$$
p_{6}=\frac{-\rho_{0} M c_{0} \bar{v}_{0} \operatorname{sgn}(y)}{2 \pi}\left(\cos ^{-1}\left(-\frac{\bar{z}}{\bar{r}_{h}}\right)-\sin ^{-1}\left(\frac{M \bar{x}-c_{0} t}{\bar{r}_{h}}\right)\right),
$$

and in region 7 at least one Heaviside function is zero for any value of $\theta$, so that $p_{7}=0$. In regions 8 and 9 , symmetry gives $p_{8}=p_{5}$ and $p_{9}=p_{3}$. Finally, in region 10, the analysis is similar to that for region 6 , with result

$$
p_{10}=\frac{-\rho_{0} M c_{0} \bar{v}_{0} \operatorname{sgn}(y)}{\pi}\left(\cos ^{-1}\left(-\frac{\bar{z}}{\bar{r}_{h}}\right)-\sin ^{-1}\left(\frac{M \bar{x}-c_{0} t}{\bar{r}_{h}}\right)-\frac{\pi}{2}\right) .
$$

These expressions show that the pressure field is continuous, except at the boundary of the Mach wedge, where there is a finite jump. The pressure field is not smooth, however: at region boundaries the gradient of the pressure is infinite. For example, near the edge of the Mach cone $\bar{R}_{h}=0$, i.e. where $\bar{r}_{h}=|\bar{z}|$, we have $p_{5} \propto \cos ^{-1}\left(-\bar{z} / \bar{r}_{h}\right) \sim$ $\sqrt{2}\left(1+\bar{z} / \bar{r}_{h}\right)^{1 / 2}$ for $\bar{z}<0$. Such a square root variation of pressure occurs at many of the boundaries.

Contours of the pressure field are shown in figure 6(b), in which the sharp angles in the contours indicate the changes in the form of the field as a wavefront is crossed. The pressure in region 4 is uninfluenced by the corner of the gust, and so is two-dimensional; hence it is constant, as for the two-dimensional top-hat gust.

\subsection{The rectangular gust}

A rectangular gust has upwash

$$
f(t-x / U, z)=v_{0} \mathrm{H}((t-x / U) / \tau,-1,1) \mathrm{H}(z / a,-1,1) .
$$

It may be written as a linear combination of four corner gusts by writing the product of the Heaviside functions as

$$
\mathrm{H}\left(t_{+}^{\prime}\right) \mathrm{H}\left(z_{+}^{\prime}\right)-\mathrm{H}\left(t_{+}^{\prime}\right) \mathrm{H}\left(z_{-}^{\prime}\right)-\mathrm{H}\left(t_{-}^{\prime}\right) \mathrm{H}\left(z_{+}^{\prime}\right)+\mathrm{H}\left(t_{-}^{\prime}\right) \mathrm{H}\left(z_{-}^{\prime}\right),
$$

where $t_{ \pm}^{\prime}=t-x / U \pm \tau$ and $z_{ \pm}^{\prime}=z \pm a$. Hence the pressure field is a linear combination of four fields of the type just calculated, after minor modifications to account for the position of the corners. The boundary of the gust produces different types of wavefront: the corners generate expanding spheres; the front and back edges generate Mach wedges and expanding cylinders; and the side edges generate cones. Hence there are many distinct regions, some of which 


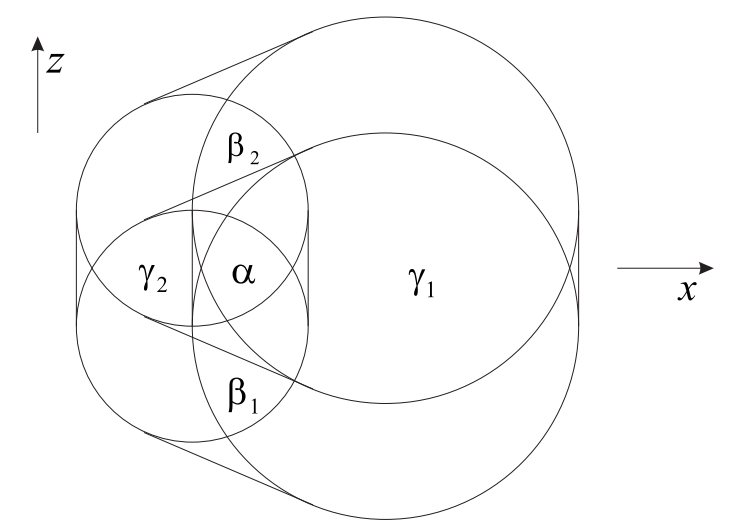

Figure 7: Section in the aerofoil plane $y=0$ of the wavefronts generated by a rectangular gust, after sufficient time has passed for the interior silent zone $\alpha$ to emerge.

exist only for a finite time, or only after a certain time, or only for a range of aspect ratios of the gust. Figure 7 shows the intersection of these wavefronts with the aerofoil plane $y=0$ for a square gust at a time shortly after the four spherical wavefronts from the corners have overlapped.

Rather than calculate the pressure in all possible regions, we shall concentrate on the four most important types of region in the far field. Three of these are labelled $\alpha, \beta$, and $\gamma$ in figure 7; a fourth type, which shrinks after a short time onto the Mach wedge, is not shown. Regions $\beta$ and $\gamma$ are each in two parts, indicated in the figure by subscripts. The region $\alpha$ lies inside all four of the spherical wavefronts generated by the corners of the gust. It exists only after sufficient time has passed, and the pressure here is identically zero. Since the region grows in time, but the surrounding regions are of fixed width, it follows that in the far field there is an almost spherical shell of impulse noise surrounding a large zero-pressure region, i.e. a zone of silence.

The shrinking region just referred to is a small region where the shell of non-zero pressure adjoins the Mach wedge. While the gust is still striking the leading edge there is a region of constant pressure near the leading edge, corresponding to region 4 of the corner gust as shown in figure 6. This constant pressure region exists outside the four spherical wavefronts generated by the corners of the gust, and as time passes the region contracts onto the Mach wedge, giving the three-dimensional equivalent of the constant-pressure pulse found in two-dimensional gusts.

The two other types of region of importance in the far field are those where only two of the spherical wavefronts overlap: these are either the two wavefronts generated at the same span position and propagating in opposite spanwise directions, giving the regions labelled $\beta_{1}$ and $\beta_{2}$ in figure 7; or the two wavefronts generated at the same time and propagating with or against the free stream, giving regions $\gamma_{1}$ and $\gamma_{1}$. The pressure in regions $\beta_{1}$ and $\beta_{2}$ is

$$
p=\frac{-\rho_{0} M c_{0} \bar{v}_{0} \operatorname{sgn}(y)}{2 \pi}\left(\sin ^{-1}\left(\frac{M \bar{x}-c_{0}(t-\tau)}{\bar{r}_{h}}\right)-\sin ^{-1}\left(\frac{M \bar{x}-c_{0}(t+\tau)}{\bar{r}_{h}}\right)\right)
$$

and the pressure in regions $\gamma_{1}$ and $\gamma_{2}$ is

$$
p_{=}=\frac{-\rho_{0} M c_{0} \bar{v}_{0} \operatorname{sgn}(y)}{2 \pi}\left(\cos ^{-1}\left(\frac{-(\bar{z}+\bar{a})}{\bar{r}_{h}}\right)-\cos ^{-1}\left(\frac{-(\bar{z}-\bar{a})}{\bar{r}_{h}}\right)\right) .
$$

In regions $\beta_{1}$ and $\beta_{2}$ the field is two-dimensional, being independent of $z$.

The evolution of the field as a function of time is shown in figure 8. In $(a)$, the gust is still striking the leading edge and the region of constant pressure is evident. In $(b)$ the gust is past the leading edge, the constant pressure region has detached from the aerofoil surface, and the rear spherical wavefronts have not overlapped. In $(c)$, the silent zone at the centre has emerged, and in $(d)$ it has grown, becoming almost spherical. The field at early times is rather complex, containing many regions with square-root type pressure fields at their boundaries, indicated by the close spacing of contours. At later times the two-dimensional regions $\beta_{1}$ and $\beta_{2}$ can be seen.

Figure 8 is for a gust which is almost square, with the aspect ratio of span to stream length $1 / \sqrt{2}$. However, the form of the generated field depends strongly on the aspect ratio: a long thin gust produces a very different sound 


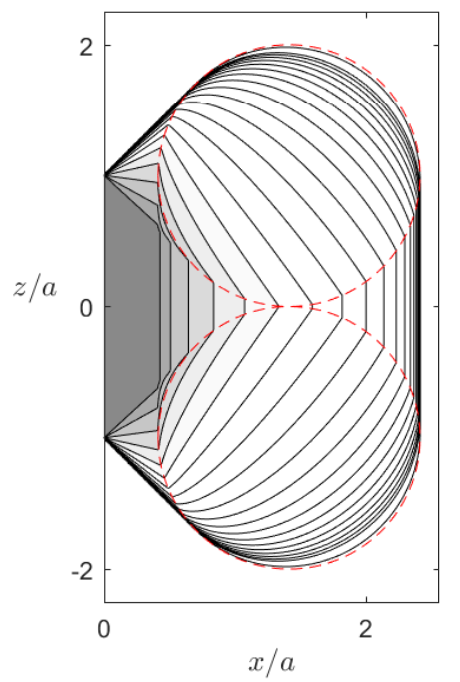

(a)

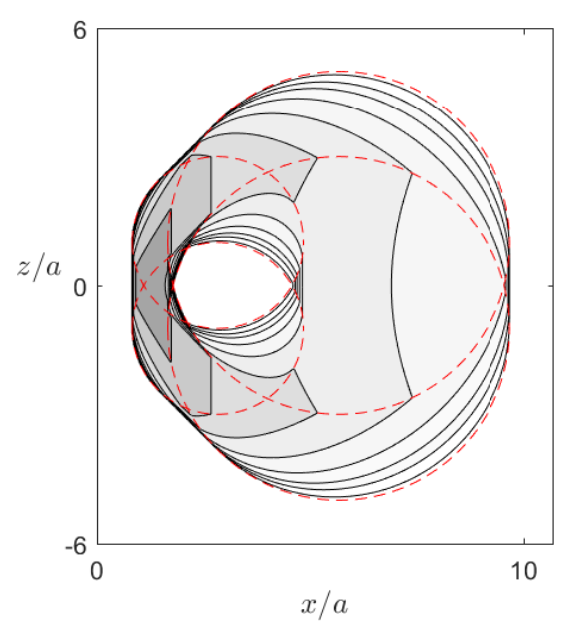

(c)

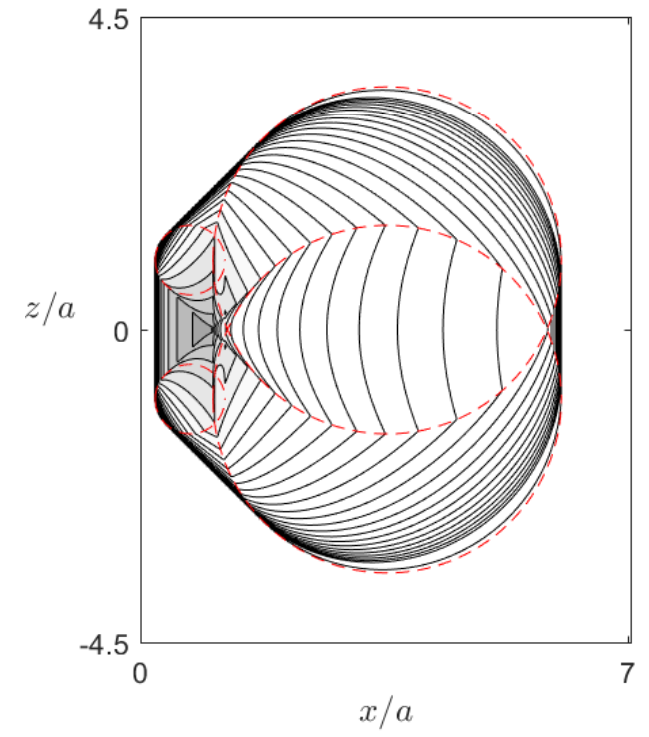

(b)

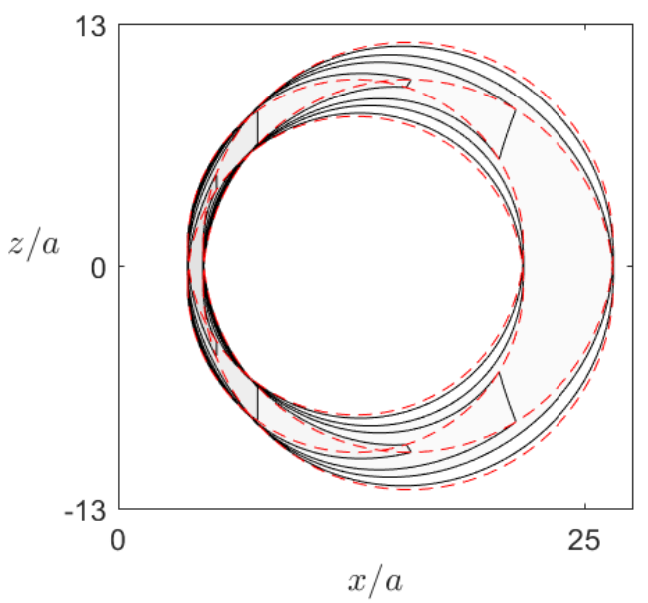

(d)

Figure 8: Contour plots in the upper surface of the aerofoil plane, $y=0$, of the pressure field produced by a rectangular gust with $c_{0} \tau=a$ for $M=\sqrt{2}$. The pressure is negative or zero, and the darkest shading indicates the most negative pressures. The dashed red circles are the wavefronts generated at the leading edge by the corners of the gust. (a) $t=0$ : the gust is still interacting with the leading edge; (b) $t=1.5 \tau$ : the gust has passed the leading edge, and the two wavefronts from the front corners have overlapped; (c) $t=3 \tau$ : all four of the wavefronts from the corners have overlapped, creating an interior silent zone; (d) $t=10 \tau$ : the silent zone is now much larger, and is surrounded by an expanding shell containing an impulsive sound field. 


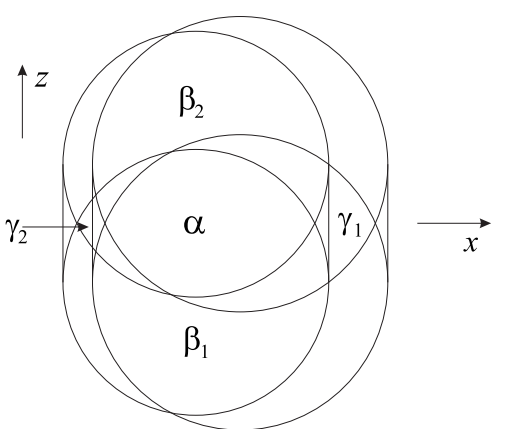

(a)

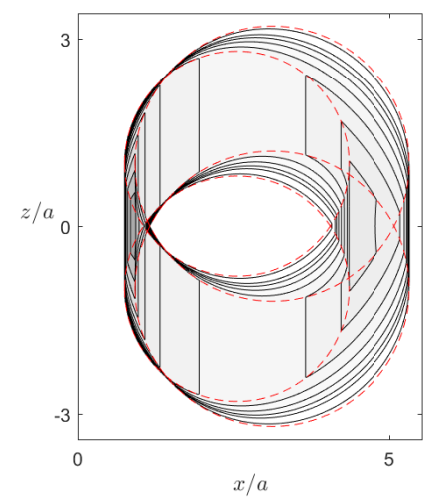

(c)

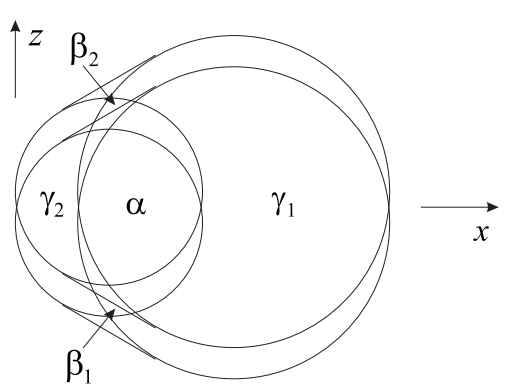

(b)

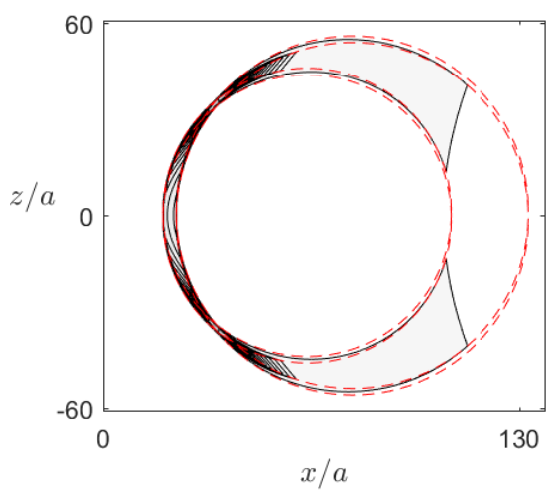

(d)

Figure 9: Effect of the aspect ratio of a rectangular gust on the form of the pressure field at time $t=10 \tau$ for $M=\sqrt{2}$. The top row shows the wavefronts in the aerofoil plane $y=0$, and the bottom row gives the pressure contours in $y=0^{+}$, with shading as for figure 8 . Plots (a) and (c) are for a short wide gust, with $c_{0} \tau=a / 5$; (b) and (d) are for a long thin gust, with $c_{0} \tau=5 a$.

field from a short wide gust. Here long and short refer to the stream direction, while thin and wide refer to the span direction. Fields for the two cases are shown in figure 9. Part $(a)$ shows the regions for a short wide gust, i.e. $c_{0} \tau \ll a$, for which the two-dimensional regions $\beta_{1}$ and $\beta_{2}$ dominate the field, whereas $(b)$ shows the regions for a long thin gust, i.e. $c_{0} \tau \gg a$, for which the regions $\gamma_{1}$ and $\gamma_{2}$ are dominant. Parts $(c)$ and $(d)$ show pressure contours for aspect-ratio parameters $c_{0} \tau / a=1 / 5$, and $c_{0} \tau / a=5$, corresponding to short wide and long thin gusts respectively. The first of these has much in common with pressure generated by the streamwise delta-function and spanwise top-hat gust of $\$ 4.1$, being almost two-dimensional except for higher pressures near the vertical plane $z=0$. By contrast, the second is similar to the pressure generated by the spanwise delta-function and streamwise top-hat gust of $\S 3.1$, except for higher pressures near the Mach cone $\bar{R}_{h}=0$; the source is effectively compact in the span direction.

\section{Gaussian smoothing}

A smoothed version of the delta-function or rectangular gust is the Gaussian gust

$$
f(t-x / U, z)=v_{0} \mathrm{e}^{-((t-x / U) / \tau)^{2} / 2} \mathrm{e}^{-(z / a)^{2} / 2},
$$

for which the Fourier transform at the leading edge $x=0$ is

$$
F(\omega, m)=2 \pi v_{0} a \tau \mathrm{e}^{-(m a)^{2} / 2} \mathrm{e}^{-(\omega \tau)^{2} / 2} .
$$


The integrals (3) and (32) for the pressure field in all space are not tractable analytically, but the far-field approximation $p \simeq p_{+}+p_{-}$obtained from (4) is

$$
p(x, y, z, t) \simeq \frac{-\rho_{0} M c_{0} \bar{v}_{0} \operatorname{sgn}(y)}{(2 \pi)^{1 / 2}} \frac{\bar{a}}{\bar{R}_{h}} \frac{1}{\left(1+\left(\bar{a} /\left(c_{0} \tau\right)\right)^{2} \tan ^{2} \bar{\theta}_{h}\right)^{1 / 2}} \sum_{ \pm} \exp \left\{-\frac{1}{2} \frac{\left(t_{ \pm} / \tau\right)^{2}}{\left(1+\left(\bar{a} /\left(c_{0} \tau\right)\right)^{2} \tan ^{2} \bar{\theta}_{h}\right)}\right\} .
$$

Here the summation is over the two values $t_{ \pm}$defined in (8). The exponential terms have maxima on the spherical wavefronts $t_{ \pm}=0$. The case $c_{0} \tau \ll a$ gives a short wide gust, with its far field concentrated near the vertical plane $z=0$; by contrast, for a long thin gust, with $c_{0} \tau \gg a$, the far field is similar to the pressure field (15) produced by a spanwise delta-function, streamwise Gaussian gust, except near the Mach cone.

\subsection{The spanwise top-hat gust}

If the streamwise delta-function in $\S 4$ is smoothed to a Gaussian, the upwash for the spanwise top-hat profile is

$$
f(t-x / U, z)=v_{0} \mathrm{e}^{-\frac{1}{2}((t-x / U) / \tau)^{2}} H(z / a,-1,1),
$$

with leading-edge Fourier transform

$$
F(\omega, m)=2^{3 / 2} \pi^{1 / 2} v_{0} \tau \frac{\sin (m a)}{m} \mathrm{e}^{-(\omega \tau)^{2} / 2} .
$$

The far-field pressure is

$$
p(x, y, z, t) \simeq \frac{-\rho_{0} M c_{0} \bar{v}_{0} \operatorname{sgn}(y)}{2^{3 / 2} \pi^{1 / 2}} \frac{\bar{a}}{\bar{R}_{h}} \frac{1}{\left(\bar{a} /\left(c_{0} \tau\right)\right) \tan \bar{\theta}_{h}}\left\{\operatorname{erf}\left(\frac{\sigma_{++}}{\sqrt{2}}\right)-\operatorname{erf}\left(\frac{\sigma_{+-}}{\sqrt{2}}\right)+\operatorname{erf}\left(\frac{\sigma_{-+}}{\sqrt{2}}\right)-\operatorname{erf}\left(\frac{\sigma_{--}}{\sqrt{2}}\right)\right\}
$$

where

$$
\sigma_{ \pm \pm}=\frac{1}{\tau}\left(t-\frac{M \bar{x}}{c_{0}} \pm \frac{\bar{R}_{h}}{c_{0}}\right) \pm \frac{\bar{a}}{c_{0} \tau} \tan \bar{\theta}_{h}
$$

and the error function erf is defined by

$$
\operatorname{erf}(s)=\frac{2}{\pi^{1 / 2}} \int_{0}^{s} \mathrm{e}^{-t^{2}} \mathrm{~d} t
$$

For a long thin gust, with $c_{0} \tau \gg a$, the far-field approximation (50) simplifies to

$$
p(x, y, z, t) \simeq \frac{-\rho_{0} M c_{0} \bar{v}_{0} \operatorname{sgn}(y)}{\pi} \frac{\bar{a}}{\bar{R}_{h}}\left(\mathrm{e}^{-\left(t_{+} / \tau\right)^{2} / 2}+\mathrm{e}^{-\left(t_{-} / \tau\right)^{2} / 2}\right),
$$

which is equivalent to (15) for a spanwise delta-function, streamwise Gaussian gust. The only difference is a factor of 2 , accounted for by the width $2 a$ of the gust (48). For a short wide gust, with $c_{0} \tau \ll a$, the field decays rapidly away from the vertical plane $z=0$; that is, it exhibits strong beaming.

\subsection{The streamwise top-hat gust}

The spanwise delta-function in $\S 3$ may be smoothed to a Gaussian, to give for a streamwise top-hat profile the upwash

$$
f(t-x / U, z)=v_{0} H((t-x / U) / \tau,-1,1) \mathrm{e}^{-(z / a)^{2} / 2},
$$

with leading-edge Fourier transform

$$
F(\omega, m)=2^{3 / 2} \pi^{1 / 2} v_{0} \tau \frac{\sin (\omega \tau)}{\omega} \mathrm{e}^{-(m a)^{2} / 2}
$$

The far-field pressure is

$$
p(x, y, z, t) \simeq \frac{-\rho_{0} M c_{0} \bar{v}_{0} \operatorname{sgn}(y)}{2^{3 / 2} \pi^{1 / 2}} \frac{\bar{a}}{\bar{R}_{h}} \sum_{ \pm}\left\{\operatorname{erf}\left(\frac{t_{ \pm} / \tau+1}{\sqrt{2}\left(\bar{a} /\left(c_{0} \tau\right)\right)\left|\tan \bar{\theta}_{h}\right|}\right)-\operatorname{erf}\left(\frac{t_{ \pm} / \tau-1}{\sqrt{2}\left(\bar{a} /\left(c_{0} \tau\right)\right)\left|\tan \bar{\theta}_{h}\right|}\right)\right\} .
$$

For a short wide gust, with $c_{0} \tau \ll a$, this simplifies to

$$
p(x, y, z, t) \simeq \frac{-\rho_{0} M c_{0} \bar{v}_{0} \operatorname{sgn}(y)}{\pi} \frac{\bar{a}}{\bar{R}_{h}} \frac{1}{\left(\bar{a} /\left(c_{0} \tau\right)\right) \tan \bar{\theta}_{h}} \sum_{ \pm} \exp \left\{-\frac{1}{2}\left(\frac{t_{ \pm} / \tau}{\left(\bar{a} /\left(c_{0} \tau\right)\right) \tan \bar{\theta}_{h}}\right)^{2}\right\},
$$

which decays rapidly away from the vertical plane $z=0$. 


\section{Conclusion}

The mathematical ideas in this paper go back to Hadamard's creation of wavefront theory for the equations of acoustics and hydrodynamics [19], and to the application of this theory to acoustic diffraction problems by Friedlander [18]. These ideas remain central in the solution of time-domain problems in acoustics. Wavefront surfaces divide space into a geometrical pattern of different regions, often in a strikingly elegant way which involves intersecting and touching cones, planes, cylinders, and spheres. The pressure field in each region has its own distinctive character, which can be conical, planar, uniform, cylindrical, or self-similar, and the transitional behaviour of the pressure across each wavefront surface may be determined analytically, leading to such possibilities as finite discontinuity; continuity but with a jump in slope; continuity but with an infinite slope on one side; or singularity on one or both sides. The neighbourhoods of points or lines where a plane touches a sphere or cylinder, for example, have their own distinctive singularities in the pressure, sensitively dependent on the direction of approach.

Although in principle the time-domain and frequency-domain approaches are equivalent, since each can be converted to the other by means of the Fourier transform combined with generalised function theory, nevertheless the approaches are different in character, not least because a single-frequency pressure field is smooth. Thus there are no surfaces of discontinuity or singularity in the frequency domain. For this reason, the canonical frequency-domain theory of supersonic leading edges is presented with its own development in the companion paper [17], in which Keller's geometrical theory of diffraction provides the unifying idea. In the present paper we have given complete details of Hadamard's and Friedlander's wavefront surfaces for a large number of impulsive sound fields produced by different types of gusts convected towards a supersonic edge, and we have provided explicit formulae for the pressure field in the different regions. The range of worked-out examples in the two papers together is comprehensive enough that it is expected to cover the main types of canonical field required in practice for a supersonic edge.

\section{References}

[1] R. Martinez, S. E. Widnall, An aeroacoustic model for high-speed, unsteady blade-vortex interaction, AIAA J. 21 (1983) $1225-1231$.

[2] R. K. Amiet, Gust response of a flat-plate aerofoil in the time domain, Quarterly J. Mech. Appl. Math. 39 (1986) $485-505$.

[3] R. K. Amiet, Airfoil gust response and the sound produced by airfoil-vortex interaction, J. Sound Vib. 107 (1986) $487-506$.

[4] R. K. Amiet, Intersection of a jet by an infinite span airfoil, J. Sound Vib. 111 (1986) 499-503.

[5] M. S. Howe, Contributions to the theory of sound production by vortex-airfoil interaction, with application to vortices with finite axial defect, Proc. Roy. Soc. A 420 (1988) 157-182.

[6] Y. P. Guo, A note on sound from the interruption of a cylindrical flow by a semi-infinite aerofoil of subsonic speed, J. Sound Vib. 128 (1989) $275-286$.

[7] C.J. Chapman, High-speed leading-edge noise, Proc. Roy. Soc. A 459 (2003) 2131-2151.

[8] C.J. Chapman, Some benchmark problems for computational aeroacoustics, J. Sound Vib. 270 (2004) $495-508$.

[9] C. J. Chapman, Sesquipoles in aeroacoustics, J. Sound Vib. 300 (2007) 1015-1033.

[10] J. E. Ffowcs Williams, Y. P. Guo, Sound generated from the interruption of a steady flow by a supersonically moving aerofoil, J. Fluid Mech. 195 (1988) 113-135.

[11] Y. P. Guo, On sound generation by a jet flow passing a semi-infinite aerofoil, AIAA paper 89-1070, Reston, VA: American Institute of Aeronautics and Astronautics (1989) 1-10.

[12] Y. P. Guo, Sound generation by a supersonic aerofoil cutting through a steady jet flow, J. Fluid Mech. 216 (1990) 193-212.

[13] N. Peake, The interaction between a steady jet flow and a supersonic blade tip, J. Fluid Mech. 248 (1993) 543-566.

[14] C. J. Powles, Noise generation by a supersonic leading edge. Part 1. General theory, J. Sound Vib. 276 (2004) $837-852$.

[15] C. J. Powles, Noise generation by a supersonic leading edge. Part 2: examples of two-dimensional sound fields, J. Sound Vib. 276 (2004) $853-868$.

[16] C. J. Powles, Supersonic leading-edge noise, PhD thesis, University of Keele (2004).

[17] C. J. Powles, C. J. Chapman, Canonical sound fields in the frequency-domain theory of supersonic leading-edge noise, Wave Motion (2019) (submitted).

[18] F. G. Friedlander, Sound Pulses, CUP, Cambridge, 1958.

[19] J. Hadamard, Leçons sur la propagation des ondes et les équations de l'hydrodynamique, Hermann, Paris, 1903. 